IBIMA Publishing

JMED Research

http://www.ibimapublishing.com/journals/JMED/jmed.html

Vol. 2014 (2014), Article ID 230675, 5 pages

DOI: $10.5171 / 2014.230675$

Research Article

\title{
Arsenic Exposure as a Cause of Persistent Absolute Eosinophilia
}

\author{
Elena Vrotsos ${ }^{1}$, Raul Martinez ${ }^{2}$, Joseph Pizzolato ${ }^{3}$, Antonio Martinez ${ }^{4}$ \\ and Vathany Sriganeshan ${ }^{1}$
}

${ }^{1}$ A.M. Rywlin, MD Department of Pathology, Mount Sinai Medical Center, Miami Beach, FL, USA

${ }^{2}$ Internal Medicine, Mount Sinai Medical Center, Miami Beach, FL, USA

${ }^{3}$ Hematology/Oncology, Mount Sinai Medical Center, Miami Beach, FL, USA

${ }^{4}$ Gastro Health Pathology Laboratory, Miami, FL, USA

Correspondence should be addressed to: Elena Vrotsos; Elena.vrotsos@msmc.com

Received: 29 October 2013; Accepted: 12 December 2013; Published: 26 February 2014

Academic Editor: Gary J. Schiller

Copyright (C) 2014 Elena Vrotsos, Raul Martinez, Joseph Pizzolato, Antonio Martinez and Vathany Sriganeshan. Distributed under Creative Commons CC-BY 3.0

\begin{abstract}
Arsenic exposure and toxicity is still a major health concern. Millions of people in Asia rely on water supply contaminated with arsenic, as mentioned in Polya (2009). Arsenic can be detected in varying concentrations in all environmental media, as shown in the research done by Mondal (2010) and Paul (2013). It is released naturally into the air by volcanoes. Arsenic compounds are used to manufacture products in the agricultural industry including insecticides, herbicides, sheep dips, wood preservatives, fungicides, and medication for eradication of tapeworms in sheep and cattle. In addition arsenic compounds were used for at least a century in the treatment of syphilis, amoebic dysentery and trypanosomaiasis. Environmental sources of arsenic are wide and include food, water, soil and air.

Arsenic toxicity can be divided into acute and chronic. The changes that can be seen with arsenic exposure are extensive and mostly non-specific like skin changes, peripheral sensorimotor neuropathy, diabetes mellitus, hypertension, pericarditis, thrombocytopenia, leukopenia, and eosinophilia.

Herein we present a case of a 32 year old woman that was referred to the hemotology/oncology service for eosinophilia. Her medical history includes asthma as a child and depression. Her medications included Wellbutrin, Abilify, Melatonin and Vitamin D. She had persistent eosinophilia for several years. Extensive work-up including B12, folate, cortisol, HIV, ESR, CRP, JAK-2 mutation, BCR-ABL, PDGFR $\alpha / \beta$ were negative. All the medications were discontinued, but her eosinophilia
\end{abstract}

Cite this Article as: Elena Vrotsos, Raul Martinez, Joseph Pizzolato, Antonio Martinez and Vathany Sriganeshan (2014), "Arsenic Exposure as a Cause of Persistent Absolute Eosinophilia," JMED Research, Vol. 2014 (2014), Article ID 230675, DOI: 10.5171/2014.230675 
persisted. Several years after her initial presentation, heavy metal urine screen was ordered and came back with high level of arsenic, 144.5 mcg per gram creatinine.

Eosinophilia is one of the non-specific symptoms that can be related to arsenic toxicity. Our case demonstrates the need for urine heavy metal screen when all other possibilities are ruled out.

Keywords: Eosinophilia, arsenic exposure, persistent absolute eosinophilia.

\section{Introduction}

Arsenic occurs in many minerals, usually in combination with sulfur or other metals, but can be found in pure form as well. Arsenic is a metalloid with properties between that of a metal and a nonmetal. Arsenic is present naturally in air, water and soil at low concentrations, but agricultural application of insecticides, herbicides or waste disposal may increase arsenic concentration to higher levels. Exposure to higher level of arsenic has been linked to multiple health problems like cardiovascular and peripheral vascular diseases, developmental abnormalities, diabetes mellitus and multiple cancers, Tchounwou (2003). Most common routes of exposure to arsenic are oral, inhalation, skin contact and parenteral route to some extent, Tchounwou (2003). High levels of arsenic have been reported in drinking water in many countries of the world including Argentina, India, Mexico, Thailand, Taiwan and Bangladesh, Tchounwou (1999). Since increase of international travel and migration, people from different countries may present with illnesses related to chronic arsenic exposure.

Well water contaminated with arsenic causes hyperpigmentation, keratosis and hypopigmentation of the skin, Mazumder (1998). Blackfoot disease, due to peripheral atherosclerosis resulting in dry gangrene, also has been associated with long-term exposure to inorganic arsenic. Tseng (1977). In the cross-sectional survey done in large population in West Bengal, India, that has been exposed to naturally occurring inorganic arsenic in the drinking water, Mazumderet al. found higher prevalence of cough, shortness of breath and chest sounds, like crepitations and/or rhonchi with increasing arsenic concentration in drinking water, Mazumder (2000). Hematologic complications of arsenic poisoning include leukopenia, granulocytopenia, profound anemia, absolute eosinophilia in addition to megaloblastic anemia, Feussner (1779).

Hypereosinophilia is defined as blood eosinophil count exceeding $0.5 \times 10^{9} / \mathrm{L}$. In many cases, hypereosinophilia is "reactive" or secondary and is due to over-production of IL- 5 by CD4+ helper T cells, Brugnoni (1996). The differential diagnosis of hypereosinophilia includes reactive response to parasitic helminth infection, adverse reaction to medications, solid tumors, mostly adenocarcinomas, lymphomas, predominantly Hodgkin's disease and T-cell lymphomas, and other eosinophil-associated syndromes, Roufosse (2010). Rarely eosinophilic expansion can be clonal resulting from mutation in the stem cell, Roufosse (2010). Heavy metal poisoning, like arsenic, rarely comes into differential diagnosis. Here we report a case of chronic arsenic exposure leading to persistent eosinophilia.

\section{Case Report}

A 32-year old woman with history of asthma and depression as a child presented to hematology/oncology services for evaluation of persistent eosinophilia on routine laboratory work. Patient's only symptom was mild abdominal pain. She denied any skin changes, diarrhea or travel history. She was originally from Mexico where she was trained as a bariatric surgeon. Her surgical history included breast augmentation and lap band procedure. Her current medications included Wellbutrin, Abilify, Melatonin and Vitamin D. Laboratory workup at 
presentation revealed WBC count of $9.9 \times 10^{3} / \mu \mathrm{L}$, hemoglobin of $13.4 \mathrm{~g} / \mathrm{dL}$, hematocrit $39.6 \%$ and platelets of $390,000 / \mu \mathrm{L}$. The differential blood count included $47 \%$ neutrophils, 29\% lymphocytes, $4 \%$ monocytes and 20\% eosinophils, (Figure 1 and Table 1). During the initial workup, stool cultures for ova and parasites were negative; cortisol level, B12 and folate were within normal limits. HIV test was negative. C-reactive protein, erythrocyte sedimentation rate, and other major electrolytes were within normal limits. Bone marrow biopsy showed normocellular bone marrow with increased eosinophils. Cytogenetic analysis of the bone marrow aspirate was normal. FISH probes for $4 \mathrm{q} 12$ (FIP1L/CHIC2/PDGFRA) and 5, 7, 8, t(8;21), $11 q 23$ (MLL), t(15;17) were normal. Atypical signal pattern was seen in the 9 (ABL/ASS) and 22(BCR) probe set, which was not consistent with clinical picture. During subsequent follow-up visits patient stopped Abilify, Melatonin, Wellbutrin, and had IUD removed. However, eosinophilia persisted. Two years after her initial visit 24-hour urine was sent for heavy metal. The patient was instructed not to consume any seafood for a least a week before the testing. The test came back positive for arsenic at $144.5 \mathrm{mcg}$ per gram creatinine. The normal urine arsenic is generally less that $10 \mathrm{mcg}$ per gram creatinine. The patient was started on Succimer, but could not tolerate oral dose and was switched to injectable Dimercaprol. Her eosinophilia is trending down and she is feeling better. She was also referred to Emory University Toxicology Program where more detailed testing was done.

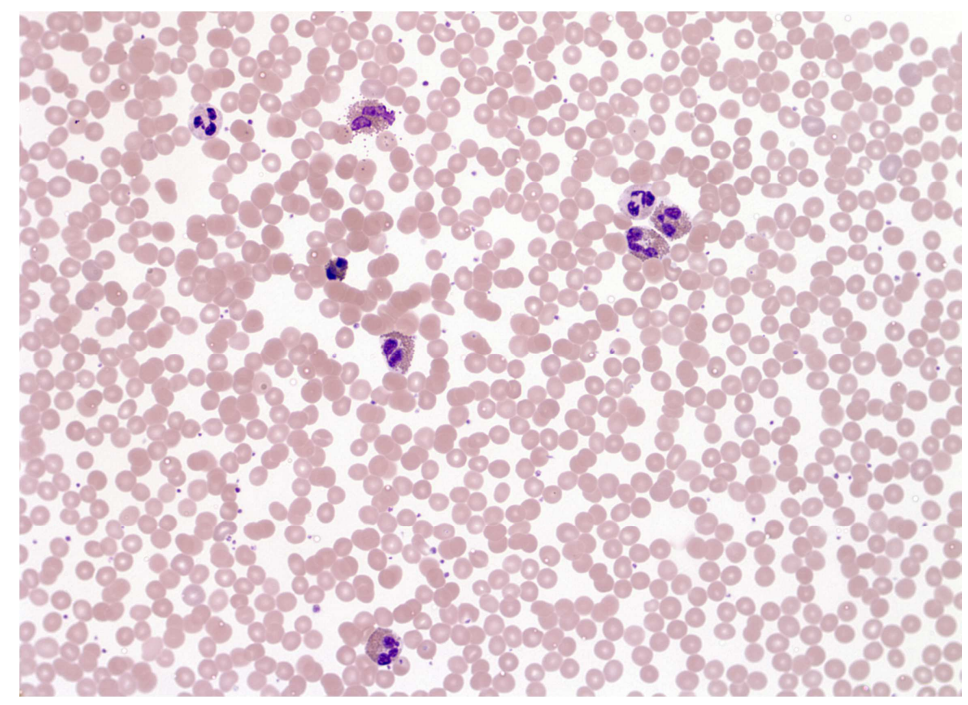

Figure 1. Peripheral Blood Smear with Increased Eosinophils (H\&E, 40x)

Table 1. Chronological Table. WBC with Percentage of Eosinophils

\begin{tabular}{|l|l|l|}
\hline Date/Lab values & WBC $\left(\times 10^{3} / \mu \mathrm{L}\right)$ & Eosinophils (\%) \\
\hline August, 2010 & 9.2 & 25 \\
\hline November, 2010 & 7.0 & 30 \\
\hline February, 2011 & 6.7 & 33 \\
\hline June, 2011 & 7.4 & $35 \%$ \\
\hline May, 2012 (after treatment) & 7.9 & $24 \%$ \\
\hline July, 2012 & 8.8 & $17 \%$ \\
\hline
\end{tabular}




\section{Discussion}

Peripheral eosinophilia can be divided into primary, secondary and idiopathic forms. In the primary form eosinophilic expansion is clonal and results from mutation in the hematopoietic stem cell. In this clonal expansion eosinophils are part of an underlying stem cell-derived myeloid malignancy and can accompany any one of the myeloid malignancies, Tefferi (2010). In the 2008 WHO classification,the two new subcategories are: chronic eosinophilic leukemia, not otherwise specified and myeloid/lymphoid neoplasms with eosinophilia and mutations involving platelet- derived growth factor receptor (PDGFR) $\alpha / \beta$ or fibroblast growth factor receptor 1 .

Idiopathic eosinophilia includes hypereosiniphilic syndrome (HES). The diagnsosis of hypereosiniphilic syndrome requires presence of a peripheral blood eosinophil count of $1.5 \times 10^{9} / \mathrm{L}$ or greater and eosinophil-mediated organ damage. The differentiation between clonal and idiopathic eosinophilia is not strict and in some instances hypereosinophilic syndrome represents an underlying myeloid malignancy, Tefferi (2010).

The main causes of secondary eosinophilia are drugs, vasculitis, nonmyeloid malignancies, and parasitic infections, especially in patients returning from endemic areas, Meltzer (2008). Allopurinol, carbamazepine and some antibiotics are among the most common drugs associated with eosinophilia, Ganeva (2008). Arsenic exposure as a cause of eosinophilia rarely comes into differential diagnosis. Acute and chronic exposure to arsenic through contamination of drinking water has been reported in many countries throughout the world including Argentina, Bangladesh, India, Mexico, Thailand and Taiwan, Tchounwou (1998).

Majority of inorganic arsenic is metabolized to monomethylarsonic acid and dimethylarsinic acid before it is excreted in the urine. Multiple theories exist as to how arsenic causes carcinogenesis. In the review by Kitchin, nine different modes of action are discussed including oxidative stress, chromosomal abnormalities, alteration in the DNA repair and DNA methylation patterns, modification of growth factors, enhancement of cell proliferation, promotion/progression, suppression of p53, and gene amplification, Kitchin (2001). Chromosomal abnormality, oxidative stress and altered growth factors as modes of arsenic carcinogenesis have supporting evidence in experimental systems, like animal and human cells, and in human tissues, Tchounwou (2003).

Exposure to arsenic has been associated with health effects in different organ systems including cardiovascular, integumentary, neurological, respiratory, gastrointestinal, genitourinary, as well as hematopoietic system.The most common hematopoietic involvement due to arsenic exposure includes anemia, leukopenia and eosinophilia, Tchounwou (2003). Thus, in a patient with persistent eosinophilia after initial workup for primary and most common secondary causes of eosinophilia, arsenic exposure should be in the differential.

The authors declare that they have no conflict of interest.

\section{References}

Brugnoni, D., Airo, P., Rossi, G., Bettinardi, A. et al. (1996). "A Case of Hypereosinophilic Syndrome is Associated with the Expansion of a CD3- CD4+ T-Cell Population Able to Secrete Large Amounts of Interleukin-5," Blood, 87(4):1416-1422

Feussner, J. R., Shelburne, J. D., Bredehoeft, S. \& Cohen, H. J. (1979). "Arsenic-Induced Bone Marrow Toxicity: Ultrastructural and Electron-Probe Analysis," Blood, 53(5):820827

Ganeva, M., Gancheva, T., Lazarova, R. et al. (2008). "Carbamazepine-Induced Drug Reaction with Eosinophilia and Systemic 
Symptoms (DRESS) Syndrome: Report of Four Cases and Brief Review," International Journal of Dermatology, 47(8):853-860

Kitchin, K. T. (2001). "Recent Advances in Arsenic Carcinogenesis:Modes of Action, Animal Model Systems, and Methylated Arsenic Metabolites," Toxicology and Applied Pharmacology, 172(3):249-61

Mazumder, D. N. G., Haque, R., Ghosh, N., De, B. K., Santra, A., Chakroborty, D. \& Smith, A. H.(1998). "Arsenic Levels in Drinking Water and the Prevalence of Skin Lesions in West Bengal, India," International Journal of Epidemiology, 27(5):871-7

Mazumder, D. N. G., Haque, R., Ghosh, N., De, B. K., Santra, A., Chakroborty, D. \& Smith, A. H. (2000). "Arsenic in Drinking Water and the Prevalence of Respiratory Effects in West Bengal, India," International Journal of Epidemiology, 29(6); 1047-52

Meltzer, E., Percik, R., Shatzkes, J., Sidi, Y. \& Schwartz, E. (2008). "Eosinophilia among Returning Travelers: A Practical Approach," The American Journal of Tropical Medicine and Hygiene, 78(5):702-709.

Mondal, D., Banerjee, M., Kundu, M., Banerjee, N. et al. (2010). "Comparison of Drinking Water, Raw Rice and Cooking of Rice as Arsenic Exposure Routes in Three Contrasting Areas of West Bengal, India," Environmental Geochemistry and Health, 32(6):463-477

Paul, S., Das, N., Bhattacharjee, M., Das, J. K. et al. (2013). "Arsenic-Induced Toxicity and Carcinogenicity: A Two-Wave Cross-Sectional Study in Arsenicosis Individuals in West Bengal, India," Journal of Exposure Science and Environmental Epidemiology, 23(2):156162
Polya, D. \& Charlet, L. (2009). "Environmental Science: Rising Arsenic Risk?," Nature Geoscience, 2:383 - 384

Roufosse, F. \& Weller, P. F. (2010). "Practical Approach to the Patient with Hypereosinophilia," Journal of Allergy and Clinical Immunology, 126(1):39-44

Tchounwou, P. B., Patlolla, A. K. \& Centeno, J. A. (2003). "Carcinogenic and Systemic Health Effects Associated with Arsenic Exposure--A Critical Review," Toxicologic Pathology, 31(6):575-88.

Tchounwou, P. B., Wilson, B. \& Ishaque, A. (1999). "Important Considerations in the Development of Public Health Advisories for Arsenic and Arsenic-Containing Compounds in Drinking Water," Reviews on Environmental Health, 14(4):211-29

Tefferi, A., Gotlib, J. \& Pardanani, A. (2010). "Hypereosinophilic Syndrome and Clonal Eosinophilia: Point-of-Care Diagnostic Algorithm and Treatment Update," Mayo Clinic Proceedings, 85(2):158-164

Tseng, W. P. (1977). "Effects and DoseResponse Relationships of Skin Cancer and Blackfoot Disease with Arsenic," Environmental Health Perspectives , 19:10919 